Colon

\section{Nutritional therapy for cancer cachexia} R F Grimble

\section{In cancers where high inflammatory stress is usual, protein rich supplements containing $n-3$ polyunsaturated fatty acids and high levels of antioxidant vitamins can reverse severe weight loss}

$\mathrm{T}$ he process of inflammation has a paradoxical effect. This is mostly due to the metabolic responses triggered by the release of the three proinflammatory cytokines, interleukin $1 \beta$ (IL-1 $\beta$ ), tumour necrosis factor $\alpha$ (TNF- $\alpha$ ), and interleukin 6 (IL-6). As an integral part of the body's response to infection and injury, these mediators release substrate, from host tissues, to support $\mathrm{T}$ and $\mathrm{B}$ lymphocyte activity, create a hostile environment for invading pathogens, via a raised body temperature and oxidant production, and initiate downregulation of the process once invasion has been defeated. ${ }^{1}$ All of these metabolic effects come at considerable cost to the host, as witnessed by the extensive tissue depletion, anorexia, and anaemia seen in severely infected and injured patients. ${ }^{2}$ However, the cost is of great biological value if recovery from infection and injury are achieved. The inflammatory process contains elements which inherently upregulate the response. Oxidants will increase proinflammatory cytokine production by activating nuclear factor $\kappa \mathrm{B}(\mathrm{NF \kappa B})$. A wide range of genes associated with the inflammatory process have $\mathrm{NF \kappa B}$ response elements in their structure. These include genes for proinflammatory cytokines and adhesion molecules. ${ }^{1}$

Tumour cells may initiate the inflammatory response as effectively as invading pathogens. However, while the inflammatory process may be effective in dealing with single malignant cells, once cancer is established the inflammatory process becomes a cause of the patient's demise, rather than a means of destroying the tumour. In addition to stimulating the cytokine mediated and hormonal aspects of the inflammatory response, tumour specific products also add to the level of inflammatory stress in the patient.

The tissue depletion that occurs during inflammation is different qualitatively to that seen during starvation and forms part of the syndrome of cancer cachexia. While starvation results primarily in fat loss, with secondary loss in muscle and visceral protein mass, cachexia results almost equally in fat and protein loss. Losses of up to $75 \%$ of body stores can occur. ${ }^{3}$ Furthermore visceral protein mass is relatively preserved. While the desire to eat is strong in starvation, in cachexia severe anorexia occurs. The precise mechanism for the appetite loss in cachexia is unclear. However, proinflammatory cytokines, raised serotoninergic activity in the hypothalamus, and leptin have been implicated. ${ }^{3}$ Chemotherapy unfortunately imposes oxidant stress on the patient, thereby providing a further boost to the inflammatory process. ${ }^{4}$

A number of approaches have been taken to improve nutritional status in cancer patients. Attempts to raise energy and protein intake by counselling have been successful, but despite improvements over a three month period, no improvement in weight, anthropometric measures, response rate, survival, or quality of life have been demonstrated. ${ }^{5}$ Disappointing results were also obtained when nutrient intake was increased by the parenteral route. The deleterious effects of parenteral nutrition (for example, increased infective complications) led the American College of Physicians, in a position paper, to conclude "parenteral nutritional support was associated with net harm, and no conditions could be defined in which such treatment appeared to be of benefit". ${ }^{3}$

While the precise mechanism(s) of cachexia is unclear it is self evident that the inflammatory process is exceedingly strong in weight losing cancer patients. Thus the patient's nutrient intake is dissipated by the hypermetabolism induced by the inflammatory state. Thus nutritional therapy, to improve survival in cancer patients, must make the inflammatory process its prime target. ${ }^{7}$ Among nutrients that may be effective in this respect are n-3 or omega-3, polyunsaturated fatty acids (n-3PUFA) and antioxidants. The former have been shown to be particularly effective anti-inflammatory agents in rheumatoid arthritis. ${ }^{8}$ Moreover, the effectiveness of n-3 PUFA in modulating the inflammatory process has been demonstrated in a diverse range of clinical situations ranging from surgery ${ }^{9}$ to adult respiratory distress syndrome. ${ }^{10}$ Fearon's group have pioneered the use of fish oil in the treatment of pancreatic cancer. The results of a number of small trials have been reported in which the oil, or the main n-3 PUFA that it contains (eicosapentaenoic acid (EPA)), has been demonstrated to reduce the high rates of weight loss in such patients. ${ }^{1112}$

As a logical extension to this work, an international, multicentre, double blind, randomised trial ${ }^{13}$ is reported in this issue of Gut [see pages 1479-86]. The study examined the effects of an n-3 PUFA and antioxidant enriched oral supplement on loss of weight and lean tissue loss in pancreatic cancer patients. In the study, 95 patients received the enriched supplement and 105 received the control diet. The former diet contained an amount of n-3 PUFA equivalent to $6 \mathrm{~g}$ fish oil per day, and vitamin $\mathrm{C}$ and $\mathrm{E}$ at over four and eight times the recommended amount for healthy subjects, respectively. This level of antioxidant vitamin supplementation was similar to that used by Pacht and colleagues $^{10}$ who successfully used a combination of n-3 PUFA and antioxidant, in an enteral formulation, to reducing severe lung inflammation in adult respiratory distress syndrome patients.

The large number of patients in the multicentre study ${ }^{13}$ permitted subgroup analysis to determine whether the dose of supplement exerted an effect. Some interesting insights were achieved by this strategy. There was a linear relationship between change in lean body weight and enrichment of plasma phospholipids with EPA, indicating that the greater the intake of n-3 PUFA, the greater the protein accretion in the patients. Furthermore, when body weight change was related to dietary protein intake, only those patients consuming the n-3 PUFA enriched formulation showed a positive relationship. In other words, a synergistic effect was obtained by consumption of protein in the presence of n-3 PUFA. Disappointingly, the authors do not mention any indices of inflammation (for example, plasma IL-6, C reactive protein) in their paper. It is not therefore possible to judge whether a concomitant reduction in inflammatory stress occurred during the time that $n-3$ PUFA was facilitating accretion of lean body mass.

In the clinical setting, n-3 PUFA are often given in immunonutrient mixtures (as in the present study ${ }^{13}$ ) and thus it is difficult to determine whether the n-3 PUFA per se are achieving the observed effect or whether there is some 
synergistic interaction within the body between the components of the clinical feed. It is likely that oxidant/antioxidant status had a part to play in the response observed in the multicentre study. ${ }^{13}$ Experimental studies have shown that antioxidants can decrease NFkKB activation and reduce muscle protein loss in animal cachexia models and during cell culture. ${ }^{14-16}$ Indeed, in a randomised double blind study on weight losing acquired immunodeficiency syndrome patients, an antioxidant-glutamine supplement increased body cell mass. ${ }^{17}$ It should be noted that glutamine, by acting as a source of glutamate, may provide one of the three amino acids (glycine, cysteine, glutamate) required for the synthesis of the key antioxidant glutathioine (GSH). Denno et al noted, in a rat model, that glutamine administered parenterally enhanced plasma and hepatic GSH concentrations. ${ }^{18}$ Cysteine acts as the rate limiting amino acid in GSH synthesis and studies in rat models show that, during low protein intakes, addition of the amino acid restores GSH to normal levels following injection with TNF- $\alpha$ or endotoxin. ${ }^{19}$ In the multicentre study, ${ }^{13}$ accretion of lean body mass in patients receiving fish oil was positively related to protein intake. While the amino acid composition of protein in the supplement is not quoted in the paper, it is feasible that it may have indirectly improved antioxidant status by providing the three necessary amino acids for GSH synthesis, thereby indirectly reducing inflammatory stress in the patients.

While there are a number of unanswered questions posed by the study it does illustrate that in cancers where high inflammatory stress is usual, protein rich supplements containing n3 PUFA and high levels of antioxidant vitamins, can reverse severe weight loss. It remains to be seen whether this effect is achieved by an anti-inflammatory mechanism and whether cancers in which cachexia is not as severe as in pancreatic cancer will respond favourably to similar nutritional therapy.

Gut 2003;52:1391-1392

Correspondence to: Professor R F Grimble, Institute of Human Nutrition, School of Medicine, University of Southampton, Southampton SO16 7PX, UK; rfg1@soton.ac.uk

\section{REFERENCES}

Grimble RF. Stress proteins in disease: metabolism on a knife edge. Clin Nutr 2001;20:469-76.

2 Grimble RF. Malnutrition and the immune response. 2. Impact of nutrients on cytokine biology in infection. Trans $\mathrm{R}$ Soc Trop Med Hyg 1994;88:615-19.

3 Barber MD, Ross JA, Fearon KC. Cancer cachexia. Surg Oncol 1999;8:133-41.

4 Kovacic P, Osuna JA jr. Mechanisms of anticancer agents: emphasis on oxidative stress and electron transfer. Curr Pharm Des 2000:6:277-309.

5 Evans WK, Nixon DW, Daly JM, et al. A randomised trial of oral nutritional support versus ad lib nutritional intake during chemo therapy for advanced colorectal and non-smallcell lung cancer. J Clin Oncol 1987:5:113-24.

6 Ovesen L, Allingstrup L, Hannibal J, et al. Effect of dietary counseling on food intake, body weight, response rate, survival, and quality of life in cancer patients undergoing chemotherapy: a prospective, randomised study. J Clin Oncol 1993: 11:2043-9.

7 Grimble RF. Nutritional modulation of immune function. Proc Nutr Soc 2001;60:389-97.

8 Calder PC. Dietary modification of inflammation with lipids. Proc Nutr Soc 2002;61:345-58.

9 Gianotti L, Braga M, Fortis C, et al. A prospective, randomized clinical trial on perioperative feeding with an arginine-, omega-3 fatty acid-, and RNAenriched enteral diet: effect on host response and nutritional status. JPEN J Parenter Enteral Nutr 1999;23:314-20.
10 Pacht ER, DeMichele SJ, Nelson JL, et al. Enteral nutrition with eicosapentaenoic acid, gamma-linolenic acid, and antioxidants reduces alveolar inflammatory mediators and protein influx in patients with acute respiratory distress syndrome. Crit Care Med 2003:31:491-500.

11 Barber MD, Ross JA, Preston T, et al. Fish oil-enriched nutritional supplement attenuates progression of the acute-phase response in weight-losing patients with advanced pancreatic cancer. J Nutr 1999;129:1120-5.

12 Wigmore SJ, Barber MD, Ross JA, et al. Effect of oral eicosapentaenoic acid on weight loss in patients with pancreatic cancer. Nutr Cancer 2000;36:177-84.

13 Fearon KCH, von Meyenfeldt MF, Moses AGW et al. Effect of a protein and energy dense n-3 fatty acid enriched oral supplement on loss of weight and lean tissue in cancer cachexia: a randomised double blind trial. Gut 2003;52:1479-86.

14 Sen CK, Khanna S, Reznick AZ, et al. Glutathione regulation of tumor necrosis factor-alpha-induced NF-kappa B activation in skeletal muscle-derived L6 cells. Biochem Biophys Res Commun 1997;237:645-9.

15 Buck M, Chojkier M. Muscle wasting and dedifferentiation induced by oxidative stress in a murine model of cachexia is prevented by inhibitors of nitric oxide synthesis and antioxidants. EMBO J 1996:15:1753-65.

16 Hyun Y, Ishiko O, Honda K, et al. Probucol decreases total body fat loss in VX2-carcinoma induced cachectic rabbits. Oncol Rep 2001;8:1309-11.

17 Shabert JK, Winslow C, Lacey JM, et al. Glutamine-antioxidant supplementation increases body cell mass in AIDS patients with weight loss: a randomized, doubleblind controlled trial. Nutrition 1999; 15:860-4

18 Denno R, Rounds JD, Faris R, et al. Glutamineenriched total parenteral nutrition enhances plasma glutathione in the resting state. J Surg Res 1996;61:35-8.

19 Hunter EA, Grimble RF. Dietary sulphur amino acid adequacy influences glutathione synthesis and glutathione-dependent enzymes during the inflammatory response to endotoxin and tumour necrosis factor-alpha in rats. Clin Sci (Lond) 1997; 92:297-305

\section{Origins of cardiac dysfunction in cirrhosis}

\section{W Jiménez, V Arroyo}

\section{Is cirrhotic cardiomyopathy a specific cardiac dysfunction of cirrhotic patients or is it induced by the hyperdynamic circulation in these patients?}

$\mathrm{T}$ he clinical course of patients with advanced liver disease is complicated by progressive impairment in circulatory function characterised by low arterial pressure, high cardiac output, and decreased systemic vascular resistance. ${ }^{1}$ Clinical and experimental investigations performed during the past two decades have shed light on the mul tiple mechanisms accounting for these disturbances. These studies have also established the pathogenic role of circulatory dysfunction in organ specific syndromes that commonly develop in cirrhotic patients, such as the hepatorenal and the hepatopulmonary syndromes. ${ }^{2}{ }^{3}$ The heart is another functionally compromised organ in cirrhotic patients. However, whether the hyperdynamic circulation, by overloading the heart, induces cirrhotic cardiomyopathy or whether this is a specific cardiac dysfunction of cirrhotic patients has been subject of extensive discussions. ${ }^{4}$

Cardiac function abnormalities in cirrhosis are clinically not apparent. However, when cardiac function is explored, a reduction in right ventricular volume, probably secondary to reduced venous return, and left ventricular dysfunction, characterised by left ventricular 
preload and volume, are observed. ${ }^{56}$ Moreover, cardiac structural abnormalities, including hypertrophy of the myocardium and increased left ventricle thickness and hence diastolic dysfunction, have also been described. ${ }^{7}$ Cirrhotic cardiomyopathy is latent, probably because of the low peripheral vascular resistance presented by these patients, which reduces cardiac afterload. The existence of an abnormal ventricular behaviour can however be unveiled during exercise or following pharmacological stress. It has been demonstrated that left ventricular end diastolic pressure increases and stroke index and left ventricular ejection fraction decrease more in cirrhotic patients than in control subjects. ${ }^{8-10}$

Impaired left ventricular performance in cirrhotic patients was initially thought to be due to the so-called alcoholic heart muscle disease, also known as alcoholic cardiomyopathy, ${ }^{11}$ because almost all earlier studies were performed in alcoholic patients. However, clear dissimilarities between alcoholic and cirrhotic cardiomyopathy exist. Firstly, depressed ventricular responsiveness has been observed in humans and rats with cirrhosis of nonalcoholic aetiology. ${ }^{12} 13$ On the other hand, alcoholic heart muscle disease is secondary to impaired contractile protein synthesis and formation of immunogenic cardiac protein acetaldehyde adducts $^{14}$ whereas clearly differentiated mechanisms are involved in the pathogenesis of cirrhotic cardiomyopathy.

Several studies have shown sympathetic and parasympathetic autonomic dysfunction in cirrhotic patients. ${ }^{15}$ Hypotheses have been raised suggesting that the origin of this abnormality could be located in the nervous system due to damage of the peripheral nerves or because of changes in endogenous neurotransmitters. ${ }^{8}{ }^{16}$ Impaired $\beta$ adrenergic signal transduction may also be an important element in the pathogenesis of cirrhotic cardiomyopathy. Experimental studies have shown decreased $\beta$ adrenergic receptor density and receptor desensitisation in cardiocytes of cirrhotic rats. ${ }^{17}$ In addition, leucocytes of cirrhotic patients also present decreased abundance of $\beta$ adrenoreceptor. ${ }^{18}$ Heart receptor and post receptor defects are supported by the demonstration of reduced function and expression of cardiac $G$ proteins in cirrhotic animals ${ }^{17}$ and impaired cardiac excitation-contraction coupling in portal hypertensive rats. ${ }^{19}$ Plasma membrane fluidity and ion channel function are impaired in cirrhosis..$^{20}$ Recently, Ward and colleagues ${ }^{21}$ described a decrease in $\mathrm{K}^{+}$current in ventricular myocytes of cirrhotic rats, which would result in a tendency to prolong QT intervals. This is in agreement with the results of Bernardi and colleagues ${ }^{22}$ showing a prolonged QT interval and other electrophysiological abnormalities in cardiac excitation and repolarisation in cirrhotic patients. Exposure of cardiac myocytes for long periods of time to endogenous substances with cardiac function inhibitory properties should also be taken into consideration. There is a wide array of cardiodepressant factors such as nitric oxide, endotoxins, endothelins, bile acids, and cytokines, that have been demonstrated to be increased in cirrhotic patients and experimental models of portal hypertension. ${ }^{123} 24$

Recently, brain natriuretic peptide (BNP), a cardiac hormone belonging to the natriuretic isopeptide family, has attracted increasing attention as an accurate marker of left ventricular dysfunction. In fact, BNP is an independent predictor of high left ventricular pressure, ${ }^{25}$ estimates left ventricular systolic dysfunction, and closely correlates with the New York Heart Association (NYHA) classification. ${ }^{26}$ The accuracy of BNP for the detection of left ventricular systolic dysfunction is similar to that of prostate specific antigen for the detection of prostate cancer, and is superior to that of mammography for breast carcinoma and Papanicolau smears for cervical cancer. ${ }^{27}$ BNP is released from cardiac ventricles in response to ventricular volume expansion and pressure overload, suggesting that BNP levels are a more sensitive and specific indicator of ventricular disorders than other natriuretic peptides. Data from heart failure investigations suggest that the increased release of BNP is a compensatory response elicited by ventricular remodelling aimed at reducing systemic pressure load hypertrophy through sodium and water diuresis. Thus BNP has become a specific marker of ventricular damage rather than just an indicator of volume overload. $^{28}$

Cardiac natriuretic peptides, namely atrial natriuretic peptide and BNP, have long been known to be elevated in cirrhotic patients as a consequence of increased cardiac release and not because of impaired hepatic extraction. ${ }^{29}{ }^{30}$ However, they have been generally regarded as markers of volume overload rather than markers of cardiac dysfunction. Recently, Wong and colleagues $^{31}$ proposed that BNP could be an indicator of cirrhotic cardiomyopathy. These authors measured cardiac natriuretic peptide levels and cardiac structural parameters in a group of 36 cirrhotic patients with and without ascites. Increased circulating levels of
BNP were related to septal thickness and left ventricular diameter at the end of diastole. ${ }^{31}$

In fact, although some authors considered cirrhotic cardiomyopathy, at best, a complication of alcoholic liver disease and, at worst, a non-existent medical invention, numerous evidence supports the concept of a specific cardiac disorder peculiar to cirrhosis. What is still an unanswered question is whether this abnormality results from the hyperdynamic circulation also present in these patients. In the current of Gut issue, Herikssen and colleagues ${ }^{32}$ use an elegant experimental approach to solve this dilemma [see pages 1511-7]. These investigators have simultaneously assessed plasma levels of BNP and total proBNP, and indicative parameters of liver and cardiac dysfunction and hyperdynamic circulation in a large group of cirrhotic patients. ProBNP is the high molecular precursor of functionally active BNP. Cleavage of proBNP is mainly located in the myocyte and results in secretion to the systemic circulation of equimolar amounts of the $\mathrm{N}$ terminal fragment of proBNP (NT-proBNP) and BNP. NT-proBNP circulates at considerable concentrations in human plasma, is stable in human blood, and is less dependent on pulsatile fluctuations, produced by postural changes or other physiological responses, than BNP. Total proBNP measurement is performed after in vitro plasma trypsinisation and it has been suggested that this is a more reliable method to assess BNP secretion as it does not depend on precursor processing. ${ }^{33}$ Confirming previous investigations, cirrhotic patients showed increased circulating levels of BNP, which paralleled the results obtained on analysing total proBNP. The most interesting finding of this study is that ventricular natriuretic peptide secretion closely correlates with indicative parameters of abnormal liver (Child score, hepatic venous pressure gradient, and serum albumin) and cardiac function (plasma volume, heart rate, and QT interval) but not with those characteristic of the hyperdynamic circulation (cardiac output and systemic vascular resistance). Therefore, these results seriously jeopardise the concept that increased BNP levels in cirrhotic patients are due to the hyperdynamic circulation. Rather, they support the fact that increased secretion of this natriuretic peptide is a consequence of ventricular dysfunction, which seems to progress in parallel with the severity of the liver disease. These findings should also stimulate further research to clearly delineate the molecular and cellular mechanisms responsible for the structural 
and functional abnormalities distinctive of cirrhotic cardiomyopathy. Identification of well defined therapeutic targets will certainly improve life quality and expectations of patients with advanced liver disease.

Gut 2003;52:1392-1394

\section{Authors' affiliations}

W Jiménez, Hormonal Laboratory, Hospital

Clinic, University of Barcelona, Spain

V Arroyo, Liver Unit, Hospital Clinic,

University of Barcelona, Spain

Correspondence to: Dr W Jiménez, Hormonal Laboratory, Hospital Clínic i Provincial, Villarroel 170, 08036 Barcelona, Spain; wjimenez@medicina.ub.es

\section{REFERENCES}

1 Arroyo V, Jiménez W. Renal and circulatory dysfunction in cirrhosis. Lights and shadows in an important clinical problem. J Hepatology 2000;32(suppl 1):157-70.

2 Arroyo V, Guevara M, Gines P. Hepatorenal syndrome in cirrhosis: pathogenesis and treatment. Gastroenterology

2002; 122:1658-76.

3 Müller C, Schenk P. Hepatopulmonary syndrome. Wien Klin Wochenschr 1999;111:339-47.

4 Blendis L, Wong F. Is there a cirrhotic cardiomyopathy? Am J Gastroenterology 2000:11:3026-8

5 Moller S, Sendergaard L, Mogelvang J, et al. Decreased right heart blood volume determined by magnetic resonance imaging: Evidence of central underfilling in cirrhosis. Hepatology 1995;22:472-8.

6 Laffi G, Barletta G, La Villa G, et al. Altered cardiovascular responsiveness to active tilting in nonalcoholic cirrhosis. Gastroenterology 1997; 113:891-8.

7 Pozzi M, Carugo S, Boari G, et al. Evidence of functional and structural cardiac abnormalities in cirrhotic patients with and without ascites. Hepatology 1997;26:1131-7.

8 Kelbaek H, Eriksen J, Brynjolf I, et al. Cardiac performance in patients with asymptomatic alcoholic cirrhosis of the liver. Am J Cardiol 1984; 54:852-5

9 Moller S, Henriksen JH. Cirrhotic cardiomyopathy: a pathophysiological review of circulatory dysfunction in liver disease. Heart 2002;87:9-15.

$10 \mathrm{Ma} \mathrm{Z}$, Lee SS. Cirrhotic cardiomyopathy: Getting to the heart of the matter. Hepatology 1996:24:451-9.

11 Fernandez-Sola J, Estruch R, Grau JM, et al. The relation of alcoholic myopathy to cardiomyopathy. Ann Intern Med 1994; 120:529-36.

12 Grose RD, Nolan J, Dillon JF, et al. Exerciseinduced left ventricular dysfunction in alcoholic and non-alcoholic cirrhosis. J Hepatol 1995;22:326-32

13 Ingles AC, Hernandez I, Garcia-Estan J, et al. Limited cardiac preload reserve in conscious cirrhotic rats. Am J Physiol 1991;260:1912-17.

14 Harcombe AA, Ramsay L, Kenna JG, et al. Circulating antibodies to cardiac proteinacetaldehyde adducts in alcoholic heart muscle disease. Clin Sci 1995;88:263-8.

15 Trevisani F, Sica G, Mainqua P, et al. Autonomic dysfunction and hyperdynamic circulation in cirrhosis with ascites. Hepatology 1999:30:1387-92.

16 Chaudhry V, Corse AM, O'Brian R, et al. Autonomic and peripheral (sensorimotor) neuropathy in chronic liver disease: a clinical and electrophysiological study. Hepatology 1999;29:1698-703

17 Ma Z, Miyamoto A, Lee SS. Role of altered $\beta$ adrenoreceptor signal transduction in the pathogenesis of cirrhotic cardiomyopathy in rats. Gastroenterology 1996;110:1191-8.

18 Gerbes AL, Remien J, Jüngst D, et al. Evidence for down regulation of $\beta_{2}$-adrenoceptors in cirrhotic patients with severe ascites. Lancet 1986;1:1409-11.

19 Zavecz JH, Bueno O, Maloney RE, et al. Cardiac excitation-contraction coupling in the portal hypertensive rat. Am J Physio 2000;279:G28-39.

20 Moreau R, Komaichi $\mathrm{H}$, Kirstetter $\mathrm{P}$, et al. Altered control of vascular tone by adenosine triphosphate-sensitive potassium channels in rats with cirrhosis. Gastroenterology 1994:106:1016-23.

21 Ward CA, Ma Z, Lee SS. Potassium currents in atrial and ventricular myocytes from a rat model of cirrhosis. Am J Physiol 1997;273: G537-44.

22 Bernardi M, Calandra S, Colantoni A, et al. Q-T interval prolongations in cirrhosis: prevalence, relationship with severity and aetiology of the disease and possible pathogenic factors. Hepatology 1998;27:28-34.

23 Jiménez W, Poo JL, Leivas A. Endothelin and systemic, renal and hepatic hemodynamic disturbances in cirrhosis. In: Arroyo V, Ginès $P$, Rodés J, eds. Ascites and renal dysfunction in liver disease. Pathogenesis diagnosis and treatment. London: Blackwell Science, 1999:291-303.

24 Ros J, Jimenez W, Lamas S, et al. Nitric oxide production in arterial vessels of cirrhotic rats. Hepatology 1995;21:554-60

25 Maeda K, Tsutamoto T, Wada A, et al. Plasma brain natriuretic peptide as a biochemical marker of high left ventricular end-diastolic pressure in patients with symptomatic left ventricular dysfunction. Am Heart $J$ 1998; 135:825-32.

26 Clerico A lervasi G, Del Chicca MG et al. Circulating levels of cardiac natriuretic peptides (ANP and BNP) measured by highly sensitive and specific immunoradiometric assays in normal subjects and in patients with different degrees of heart failure. J Endocrine Invest 1998;21:170-9.

27 McDonagh TA, Robb SD, Murdoch DR, et al. Biochemical detection of left-ventricular systolic dysfunction. Lancet 1998:351:9-13.

28 Dhingra H, Roongsritong C, Kurtzman NA. Brain natriuretic peptide: role in cardiovascular and volume homeostasis. Semin Nephrol 2002;22:423-37.

29 Gines $\mathbf{P}$, Jimenez W, Arroyo V, et al. Atrial natriuretic factor in cirrhosis with ascites: plasma levels, cardiac release and splanchnic extraction. Hepatology 1988;8:636-42.

30 La Villa G, Romanelli RG, Casini Raggi V, et al. Plasma levels of brain natriuretic peptide in patients with cirrhosis. Hepatology 1992;16:156-61.

31 Wong F, Siu S, Liu P, et al. Brain natriuretic peptide: is it a predictor of cardiomyopathy in cirrhosis. Clin Sci 2001;101:621-8.

32 Henriksen JH, Gøtze JP, Fuglsang S, et al. Increased circulating pro-brain natriuretic peptide (proBNP) and brain natriuretic peptide (BNP) in patients with cirrhosis: relation to cardiovascular dysfunction and severity of disease. Gut 2003;52:1511-7.

33 Goetze JP, Kastrup J, Pedersen F, et al. Quantification of pro-B-type natriuretic peptide and its products in human plasma by an analysis independent precursor processing. Clin Chem 2002;48: 1035-42.

\section{Transplanting the genetic susceptibility to Crohn's disease M H Holtmann, M F Neurath}

\section{Susceptibility to Crohn's disease may be transferred via haematopoietic stem cells, highlighting the pivotal role of genetic factors in the pathogenesis of Crohn's disease}

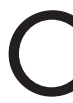

rohn's disease (CD) is one of the two most common forms of inflammatory bowel disease (IBD). The prevalence of $\mathrm{CD}$ has increased in Western countries over the past decades and mainly young patients are affected, with a peak incidence between 15 and 35 years. ${ }^{1}$ The aetiology of IBD is still unclear and should be considered as multifactorial according to recent studies. ${ }^{2}$ Genetic factors seem to play a pathogenic role as well as environmental, infectious, and immunological factors. All of these different aetiological aspects are reconciled in a paradigm, in which $\mathrm{CD}$ could result from disturbances of the intestinal barrier and pathological activation of the intestinal immune response towards luminal bacterial antigens in individuals with genetic susceptibility.

Immunological key players for the pathogenesis of $\mathrm{CD}$ have been identified, including cellular components such as lamina propria macrophages and CD4+ T lymphocytes as well as cytokines such as tumour necrosis factor $\alpha$ (TNF$\alpha$ ), interleukin (IL)-6, IL-12, IL-18, and others. ${ }^{1-3}$ Identification of these pathogenetically relevant factors has been greatly facilitated by the availability of appropriate animal models, in particular genetically engineered knockout mice or transgenic mice, respectively. When 
SCID mice lacking functional B cells and $\mathrm{T}$ cells are reconstituted with a special subset of CD4+ T helper cells expressing the surface markers CD45Rbhigh or CD62L, they develop chronic colitis. ${ }^{4}$ These $\mathrm{T}$ helper cell subsets are thought to differentiate preferentially towards Thl cells in the host producing those proinflammatory cytokines that are involved in the pathogenesis of CD, such as TNF- $\alpha .^{56}$ To date, however, clinical and experimental evidence for the role of distinct mononuclear cell populations has been limited. There are some reports on long term remission of CD after bone marrow transplantation. ${ }^{7}$ Furthermore, human immunodeficiency virus (HIV) associated decrease in CD4+ $\mathrm{T}$ helper cell number seems to induce clinical remission of $\mathrm{CD}^{8}{ }^{8}$ In addition, it has recently been shown that the immunosuppressive drug of choice for the long term treatment of CD, azathioprine, promotes apoptotic cell death of lamina propria CD4+ $\mathrm{T}$ helper cells and one effect of the anti-TNF antibody Infliximab is the rapid induction of apoptosis of peripheral blood monocytes and lamina propria T cells. ${ }^{9} 10$

In their case report in this issue of Gut, Sonwalkar and colleagues ${ }^{11}$ report on a patient with Hodgkin's lymphoma who developed fulminant colitis following non-myeloablative allogeneic stem cell transplantation (ASCT) [see pages 1518-21]. Although the clinical course with sudden onset of severe bloody diarrhoea and pancolitis sparing the terminal ileum were atypical, the colitis was classified as Crohn's colitis based on the histological findings of patchy transmural inflammation and the presence of non-caseating epitheloid granuloma. In addition, thorough diagnostic testing ruled out tuberculosis, graft versus host disease, neutropenic colitis, vasculitis, HIV, herpes simplex virus, or cytomegalovirus colitis as potential differential diagnoses. This report is remarkable in so far as it strongly suggests-without proving definitely-for the first time directly in the human system that susceptibility to $\mathrm{CD}$ can be transferred via haematopoietic stem cells. This report might thus be considered as a proof of principle for the pivotal role of genetic factors in the pathogenesis of CD.

An aetiological role of genetic factors has long been discussed based on family and ethnic studies. The first molecular genetic evidence was provided by large linkage analyses by microsatellites, suggesting relevant genetic loci on chromosome 5 (IBD5), chromosome 6 (IBD3), and chromosome 16 (IBD1). ${ }^{12}$ The IBD5 locus comprises a cluster of genes coding for cytokine genes involved in Thl/ Th2 differentiation, and single nucleotide polymorphisms are associated with susceptibility to CD. Finally, certain HLA haplotypes located at IBD3 have been suggested to confer a slightly increased overall risk for the development of CD.

The recent cloning of the NOD2/ CARD15 gene on the gene locus IBD1 and the identification of a large number of different NOD2 mutations in a subgroup of patients with $\mathrm{CD}^{13}{ }^{14}$ has raised new interest in genetics in CD. Most mutations are localised in a structurally characteristic $\mathrm{C}$ terminal domain of the NOD2 protein that resembles bacterial lipopolysaccharide binding toll-like receptors. In vitro studies showed that NOD2/CARD15 activates the transcription factor nuclear factor $\kappa \mathrm{B}$. There is evidence that NOD2/CARD15 is expressed in monocytes and intestinal epithelial cells. As a potential intracellular receptor for bacterial components, NOD2/CARD15 may be involved in the early innate immune response (including defensin production) that induces the physiological state of tolerance towards bacterial antigens from the gut lumen. ${ }^{15}$ This concept might help to explain why inactivation of NOD2/ CARD15 increases susceptibility to CD. Finally, NOD2/CARD15 mutations in CD correlate with the development of ileal and fibrostenotic forms of CD. ${ }^{16}$

To support the idea that susceptibility for CD has been transferred by ASCT, the authors performed a detailed genetic analysis of the CD susceptibility loci in the patient who developed CD after ASCT and in the donor. ${ }^{11}$ This included the NOD2/CARD15 gene on the IBDI locus, including the $5^{\prime}$ UTR (chromosome 16) HLA haplotypes on the IBD3 locus (chromosome 6), with special focus on non-classical HLA class III gene haplotypes and three single nucleotide polymorphisms at the IBD5 locus.

Although the screening was negative for all 30 NOD2/CARD15 mutations described, there was a change in a $5^{\prime}$ UTR polymorphism of the NOD2/ CARD15 gene at position -33 between donor and recipient. The donor and post ASCT recipient were homozygous for a $\mathrm{T}$ allele that may be associated with $\mathrm{CD}$, while the pre ASCT recipient was homozygous for the wild-type $G$ allele.

High resolution molecular typing confirmed that donor and pre ASCT recipient DNAs were matched for most of the HLA class I and II haplotypes except for HLA-DPBI and HLA-B where a novel allelic variant was identified in the recipient. However, genotyping for 320 single nucleotide polymorphisms in 24 non-classical HLA class III genes at IBD3 between HLA-E and TAPBP revealed significant mismatches at several sites, including MICB, TNF, HSP70, NOTCH4, and LMP2 and a double haplotype mismatch at LMP7.
The 8.1 HLA haplotype previously associated with CD was not found.

The authors conclude correctly that the findings of the HLA class mismatches at IBD3 and the CD associated polymorphism of the 5' UTR of NOD2/ CARD15 do not prove that these genetic variations are the underlying cause for an adoptive transfer of genetic susceptibility to CD from donor to recipient via ASCT. Nevertheless, their findings make the idea likely. This case thus nicely illustrates the meaning of the multifactorial pathogenesis of CD. The presence of genetic susceptibility factors by itself is not sufficient to elicit clinical CD as the donor never had symptoms of colitis. Development of clinical CD requires the coincidence of genetic susceptibility and a special microenvironment in the gut, depending on alterations of the intestinal epithelium and/or the intestinal flora. It may be speculated whether conditioning chemotherapy, including fludarabine and melphalan, altered the intestinal epithelium or whether the long term antibiotic and immunosuppressive therapy post ASCT might have altered the patient's intestinal microenvironment such that colitis could develop from susceptibility factors carried over from the stem cell donor to the recipient.

Starting from the present case of a probable adoptive transfer of $\mathrm{CD}$, the authors discuss whether ASCT donor selection should include screening for IBD. They suggest that formal questioning about IBD should be included during ASCT donor ascertainment. However, given the current paradigm of IBD having a multifactorial genesis, ${ }^{17}$ what consequence would such a screening have? In light of the efforts necessary, using word wide data bases to identify appropriate HLA matched donors for ASCT in a timely manner, would one really decline a potential donor only because of a family history of IBD? Given the weak correlation of most IBD linked genes with clinical development of the disease, should volunteers be kept from stem cell donation because of genetic susceptibility for IBD? Larger studies are warranted, including formal questioning about IBD in the family history of stem cell donors and recipients as proposed by the authors, complemented by molecular screening for relevant gene loci such as NOD2 and prospective follow up of these patients. This way, empiric data might be generated to address this particular question.

However, the issue raised by the authors should be put into a broader perspective. The molecular approach in medical research has led to the identification of numerous genes as potentially 
relevant for disease and their number is increasing rapidly. Apart from other ethical aspects, the impact for transplant medicine has to be discussed. How should the knowledge be handled about genes in organ donors and recipients that are implicated in the pathogenesis of disease in a non-monogenetic fashion with varying penetrance?

With regard to the present case the answer is easy: for the time being our understanding of the role of genetics in IBD is to preliminary to justify the exclusion of a patient with a positive family history for IBD or with proven genetic susceptibility factors from stem cell donation. In more general terms, however, there will be increasing need for debate of this issue in the future.

Gut 2003;52:1394-1396

\section{....................}

\section{Authors' affiliations}

M H Holtmann, M F Neurath, 1st Department of Medicine, Johannes Gutenberg-University, Mainz, Germany

Correspondence to: $\operatorname{Dr} M$ F Neurath, Department of Medicine, Johannes-GutenbergUniversity, Langenbeckstrasse 1, 55131
Mainz, Germany;

neurath@1-med.ḱlinik.uni-mainz.de

\section{REFERENCES}

1 Duchmann R, Zeitz M. Crohn's disease. In: Ogra P, Strober W, eds. Handbook of mucosal immunology. San Diego: Academic Press, 1999:1005

2 Shanahan F. Crohn's disease. Lancet 2002;359:62-9.

3 Atreya R, Mudter J, Finotto S, et al. Blockade of interleukin 6 trans signaling suppresses T-cell resistance against apoptosis in chronic intestinal inflammation: evidence in Crohn disease and experimental colitis in vivo. Nat Med 2000;6:583-8.

4 Powrie F, Leach MW, Mauze S, et al. Inhibition of Th1 responses prevents inflammatory bowel disease in scid mice reconstituted with CD45RBhi CD4+ T cells. Immunity 1994;2:553-62

5 Holtmann MH, Douni E, Schutz M, et al. Tumor necrosis factor-receptor 2 is upregulated on lamina propria mononuclear cells in Crohn's disease and promotes experimental colitis in vivo. Eur J Immunol 2002;32:3142-51.

6 Neurath MF, Fuss I, Kelsall BL, et al. Antibodies to interleukin 12 abrogate established experimenta colitis in mice. J Exp Med 1995; 182:1281-90.

7 Lopez-Cubero SA, Sullivan KM, McDonald GB. Course of Crohn's disease after allogenic bone marrow transplantation. Gastroenterology 1998; 114:433-40.

8 James SP. Remission of Crohn's disease after human immunodeficiency virus infection. Gastroenterology 1988;95:1667-9.
9 ten Hove T, van Montfrans C, Peppelenbosch MP, et al. Infliximab treatment induces apoptosis of lamina propria T lymphocytes in Crohn's disease. Gut 2002;50:206-11.

10 Tiede I, Fritz G, Strand S, et al. CD28-dependent Racl activation is the molecular target of azathioprine in primary human CD4+ T lymphocytes. J Clin Invest 2003; 111:1133-45.

11 Sonwalkar SA, James RM, Ahmad T, et al. Fulminant Crohn's colitis after allogeneic stem cell transplantation. Gut 2003;52:1518-21.

12 Curran ME, Lau KF, Hampe J, et al. Genetic analysis of inflammatory bowel disease in a large European cohort supports linkage to chromosomes 12 and 16. Gastroenterology 1998; 115:1066-71

13 Ogura Y, Bonen DK, Inohara N, et al. A frameshift mutation in NOD2 associated with susceptibility to Crohn's disease. Nature 2001;411:603-6

14 Hugot JP, Chamaillard M, Zouali $\mathrm{H}$, et al. Association of NOD2 leucine-rich repeat variants with susceptibility to Crohn's disease. Nature 2001;411:599-603.

15 Hisamatsu T, Suzuki M, Reinecker HC, et al. CARD15/NOD2 functions as an antibacterial factor in human intestinal epithelial cells. Gastroenterology 2003; 124:993-1000.

16 Abreu MT, Taylor KD, Lin YC, et al. Mutations in NOD2 are associated with fibrostenosing disease in patients with Crohn's disease. Gastroenterology 2002;123:679-88.

17 Schölmerich J. New developments in aetiological mechanisms of inflammatory bowe disease. Eur J Gastroenterol Hepatol 2003;15:585-6. 